\title{
Scalable Electrochemical Synthesis of Novel Biogenic Silver Nanoparticles and Its Application to High-Sensitive Detection of 4-Nitrophenol in Aqueous System
}

\author{
Van-Tuan Hoang $\mathbb{D}^{1}$, Ngo Xuan Dinh $\mathbb{D}^{1},{ }^{1}$ Tuyet Nhung Pham $\mathbb{D}^{1},{ }^{1}$ Tran Vinh Hoang $\mathbb{D}{ }^{2}$ \\ Pham Anh Tuan, ${ }^{3,4}$ Tran Quang Huy $\mathbb{0},{ }^{1,5}$ and Anh-Tuan Le $\mathbb{C}^{1,3}$ \\ ${ }^{1}$ Phenikaa University Nano Institute (PHENA), Phenikaa University, Hanoi 12116, Vietnam \\ ${ }^{2}$ School of Chemical Engineering, Hanoi University of Science and Technology, 01 Dai Co Viet Street, Hai Ba Trung District, \\ Hanoi 10000, Vietnam \\ ${ }^{3}$ Faculty of Materials Science and Engineering, Phenikaa University, Hanoi 12116, Vietnam \\ ${ }^{4}$ Vicostone Joint Stock Company, Phenikaa Group, Hanoi 12116, Vietnam \\ ${ }^{5}$ Faculty of Electric and Electronics, Phenikaa University, Hanoi 12116, Vietnam
}

Correspondence should be addressed to Van-Tuan Hoang; tuan.hoangvan@phenikaa-uni.edu.vn and Anh-Tuan Le; tuan.leanh@phenikaa-uni.edu.vn

Received 4 November 2020; Revised 30 December 2020; Accepted 6 January 2021; Published 18 January 2021

Academic Editor: Xinyu Zhang

Copyright (c) 2021 Van-Tuan Hoang et al. This is an open access article distributed under the Creative Commons Attribution License, which permits unrestricted use, distribution, and reproduction in any medium, provided the original work is properly cited.

\begin{abstract}
This study reports a scalable green electrochemical synthesis of novel biogenic silver nanoparticles colloid (biogenic AgNPs) in large scale up to 5 liters using the bulk silver bar and the green tea leaves (Camellia sinensis) extract (GTE) as reducing agent during the electrochemical process. Under a direct-current voltage source, the biomolecules in GTE can release electrons to promote the reducing process of $\mathrm{Ag}^{+}$to $\mathrm{Ag}^{0}$. More interestingly, the formation of the intermediate complex helps to cap on the nanoparticles, which leads to stabilizing AgNPs. The as-synthesized biogenic AgNPs with the size of $34 \mathrm{~nm}$ exhibit the outstanding electrochemical properties due to the presence of biomolecules on the biogenic AgNPs surface, which facilitates the effective attaching of AgNPs on the carbon surface of the screen-printed carbon electrode (SPE) through the formation of the strong $\mathrm{C}-\mathrm{O}$ coordinate bonds between $\mathrm{O}$ atom of oxygen functional groups and $\mathrm{C}$ atom of SPE. The electrochemical properties of the biogenic AgNPs-modified SPE are enhanced significantly in comparison with bare SPE and pure AgNPs-SPE. The biogenic AgNPs-SPE is applied successfully to the detection of 4-nitrophenol (4-NP). The electrochemical sensor using biogenic AgNPs can reliably detect 4-NP in the linear range from 0.1 to $25 \mu \mathrm{M}$ with the sensitivity about $6.69 \mu \mathrm{A} \mu \mathrm{M}^{-1} \mathrm{~cm}^{-2}$. The present work reveals, as the greener synthesis method with ultra-large scalable ability, high purity, and excellent electrochemical properties of biogenic AgNPs is very promising for technological applications in high-sensitive electrochemical chemosensors, nanopharmaceuticals, and other fields.
\end{abstract}

\section{Introduction}

AgNPs are one of the nanomaterials possessing the highest degree of commercialization owing to the full range of potential applications in sensors, biomedical imaging, disinfection agents, healthcare products, cleaning agents, food storage, textile coatings, and medical devices [1-3]. The synthetic methods of AgNPs can be divided into chemical, physical, and biological synthesis [3], in which biosynthesis has attracted increasing interest in the production of biogenic AgNPs due to its simplicity, low cost, and usage of environmentally friendly materials. Instead of using chemical compounds as reducing agents, this approach employs natural biological agents, including plants, algae, bacteria, yeast, and fungi. These agents not only play roles to reduce silver ions $\left(\mathrm{Ag}^{+}\right)$to metallic silver $\left(\mathrm{Ag}^{0}\right)$ but also stabilize the formed 
AgNPs during the synthetic process. Plant extracts, more namely, green tea extract, have been widely used for the synthesis of AgNPs because it is a rich source of polyphenolic compounds $[4,5]$. Moreover, the GTE has also been employed in cosmetic and therapeutic applications due to its safety and environmentally friendly. Unfortunately, despite having many unique advantages, the traditional methods used green tea extract have been still limited by requiring time-consuming and the difficulty in scaled-up production.

Meanwhile, the electrochemical method has been recently considered as a promising approach for the straightforward synthesis of pure AgNPs with high quality, large scale, and low cost $[6,7]$. In this technique, the bulk silver electrodes are the major pure precursor for the formation of AgNPs. A bulk silver electrode is anodically dissolved to create $\mathrm{Ag}^{+}$, and then, they are reduced to $\mathrm{Ag}^{0}$ under the presence of metal salts in the electrolyte at the cathode. Based on the advantages and disadvantages of both biosynthesis method and electrochemical method, in this work, we propose a scalable green electrochemical process to rapidly synthesize biogenic AgNPs in large scale up to 5 liters using the bulk silver bar and the GTE as reducing agent to replace metal salts in the normal electrochemical method which is aimed at promoting synthetic efficiency in the short time and safety of the product. Besides, with the presence of biomolecules in extract solution, this reduces the large amounts of aggregation of silver on the surface of the cathode site and enhances their stability. Moreover, to analyze the applicability of the formed product, the biomolecule-based functionalized AgNPs are considered to be the critical factor of the electrode modification for the functional electrochemical sensors due to the unique features such as good electrocatalytic properties and high conductivity, as well as compatibility with other biomolecules $[8,9]$. We have studied potential electrochemical properties of biogenic AgNPs as a signal translation probe towards the sensitive detection of 4-nitrophenol (4-NP), one of the hazardous substances causes headache, drowsiness, nausea, and cyanosis for humans, which is found into the environment as a side product in the production process of pharmaceuticals, pesticides, and dyes [10-12]. The formation mechanism and excellent electrochemical properties of biogenic AgNPs have been discussed in detail.

\section{Experimental Procedures}

2.1. Synthesis of Biogenic AgNPs by Modified Electrochemical Method. The proposed method for preparing biogenic AgNPs is based on the green electrochemical process using the inexpensive Ag metal bares as two electrodes. The GTE solution was prepared by boiling $1 \mathrm{~g}$ green tea leaves in $100 \mathrm{~mL}$ distilled water for $10 \mathrm{~min}$. This extract solution was diluted to $500 \mathrm{~mL}$ at room temperature. Then, two parallel silver electrodes were immersed in a $500 \mathrm{~mL}$ glass beaker, which was filled extract solution under the magnetic stirring condition and was connected to the DC voltage source. The electrolysis was carried out with various supplied voltages from $4 \mathrm{~V}$ to $14 \mathrm{~V}$ at room temperature and under magnetic stirring for $30 \mathrm{~min}$. The obtained biogenic AgNP solution was stored under ambient conditions in plastic containers.
Scaled-up production of the biogenic AgNPs has successfully performed using two large silver electrodes $(L \times W \times H=45 \times 4 \times 0.6 \mathrm{~cm})$ and $6 \mathrm{~L}$ cylinder glass vase. The GTE solution was prepared by boiling $10 \mathrm{~g}$ green tea leaves in 1 liter distilled water for $10 \mathrm{~min}$, filtering and then diluted to $5 \mathrm{~L}$ at room temperature. Electrolysis was carried out under continuous stirring for $60 \mathrm{~min}$ at room temperature and at a voltage of $12 \mathrm{~V}$. The obtained product was stored under ambient conditions.

2.2. Characterization of Biogenic AgNPs. The biogenic AgNP formation was confirmed by an ultraviolet-visible (UV-vis) spectrophotometry (HP 8453 spectrophotometer). The crystalline structure of biogenic AgNPs was analyzed by a Bruker D5005 X-ray Diffractometer using $\mathrm{Cu} \mathrm{K} \alpha$ radiation $(\lambda=0.154056$ $\mathrm{nm}$ ) under a voltage of $40 \mathrm{kV}$. The morphology of biogenic AgNPs was studied by a JEOL JEM 1010 transmission electron microscope (TEM) at an accelerating voltage of $80 \mathrm{kV}$.

2.3. Electrochemical Measurements. The screen-printed carbon electrodes (SPEs), DRP-110-U75 purchased from Metrohm, were carefully polished with $0.05 \mu \mathrm{m} \mathrm{Al}_{2} \mathrm{O}_{3}$ and were thoroughly cleaned by sonication in distilled water and ethanol. Biogenic AgNPs-SPEs were prepared by depositing three drops ( $1 \mu \mathrm{L}$ each) of the biogenic AgNP solution onto a bare SPE. These electrodes were dried in air at room temperature. For comparison, pure AgNPs were synthesized by an electrochemical method as described above using $\mathrm{NaBH}_{4}$ as both electrolyte and reductant agents instead of GTE solution. Pure AgNPs-SPEs were prepared with a similar procedure. Electrochemical measurements were performed using PalmSens 3 analyzer, controlled by a PC running PSTrace software version 4.8.1. The cyclic voltammetry $(\mathrm{CV})$ was conducted from $-0.3 \mathrm{~V}$ to $0.6 \mathrm{~V}$ with the scan rate of $50 \mathrm{mV} \mathrm{s}^{-1}$. Electrochemical impedance spectroscopy (EIS) was recorded in $0.1 \mathrm{~mol} \mathrm{~L}^{-1} \mathrm{KCl}$ solution, where the frequency range is from $0.01 \mathrm{~Hz}$ to $10 \mathrm{kHz}$ with a signal amplitude of $5 \mathrm{mV}$.

\section{Results and Discussion}

3.1. The Characterization of As-Synthesized Biogenic AgNPs. The biogenic AgNPs formation was initially analyzed by optical properties using UV-visible spectroscopic analysis. Figure 1(a) shows the UV-vis absorption spectra of synthesized samples under different voltages. It can be seen that no absorption peak was found for the samples synthesized at the voltage of $4 \mathrm{~V}, 6 \mathrm{~V}, 8 \mathrm{~V}$, and $10 \mathrm{~V}$, respectively. In contrast, at the voltage of $12 \mathrm{~V}$ and $14 \mathrm{~V}$, they exhibited strong absorption peaks at $437 \mathrm{~nm}$ and $443 \mathrm{~nm}$, respectively, due to the surface plasmon resonance (SPR) effect of AgNPs [1, 2]. In our present experiment, it demonstrates that AgNPs did not form when the applied voltage was $<12 \mathrm{~V}$. On the other hand, it is also noted that the absorption band was shifted to longer wavelengths for biogenic AgNPs synthesized at $14 \mathrm{~V}$ as compared to the sample at $12 \mathrm{~V}$. This shift can be attributed to the formation of the AgNPs with larger sizes in the solution. It can be seen that the applied voltage strongly affects the formation and growth of biogenic AgNPs. 


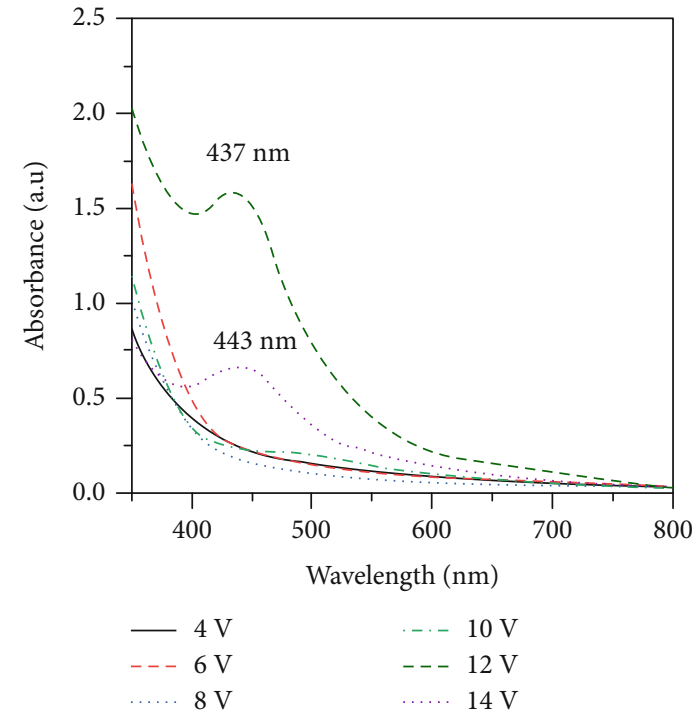

(a)

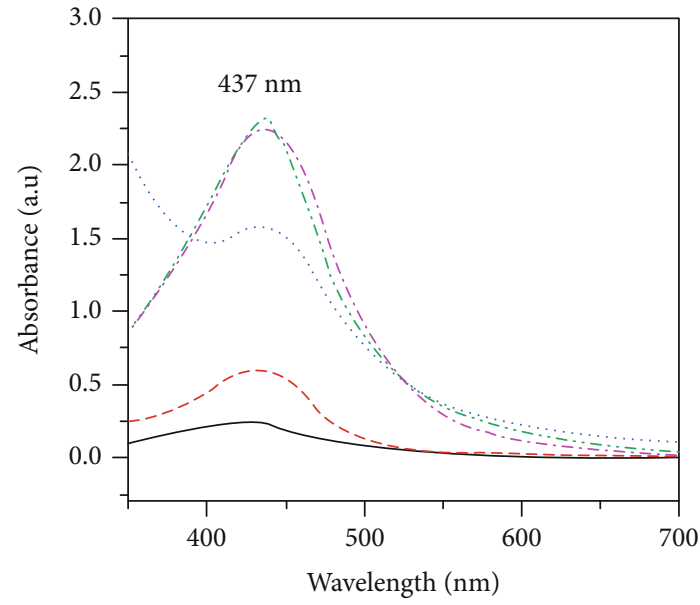

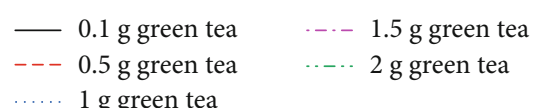

(b)

FIGURE 1: UV-vis spectra of the synthesized biogenic AgNPs corresponding to the different supplied voltages from (a) $4 \mathrm{~V}$ to $14 \mathrm{~V}$ and different amounts of green tea from (b) $0.1 \mathrm{~g}$ to $2 \mathrm{~g}$.

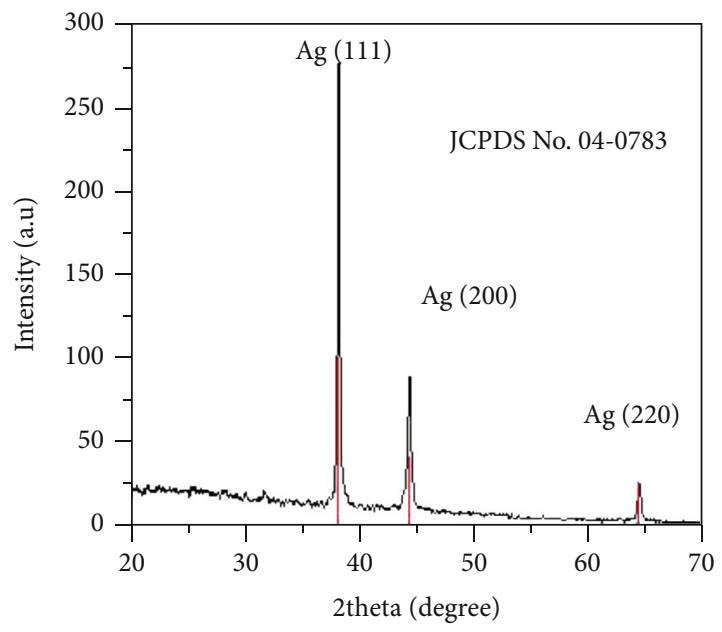

(a)

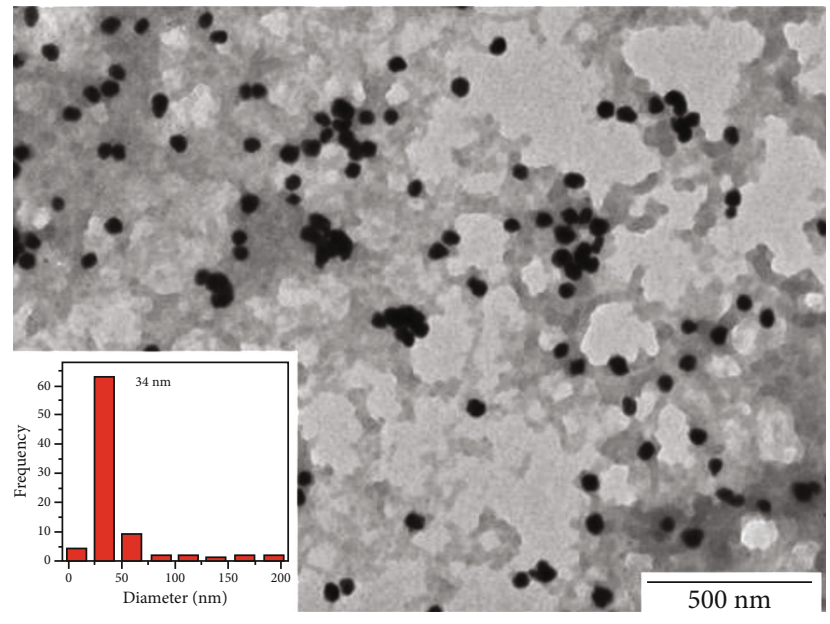

(b)

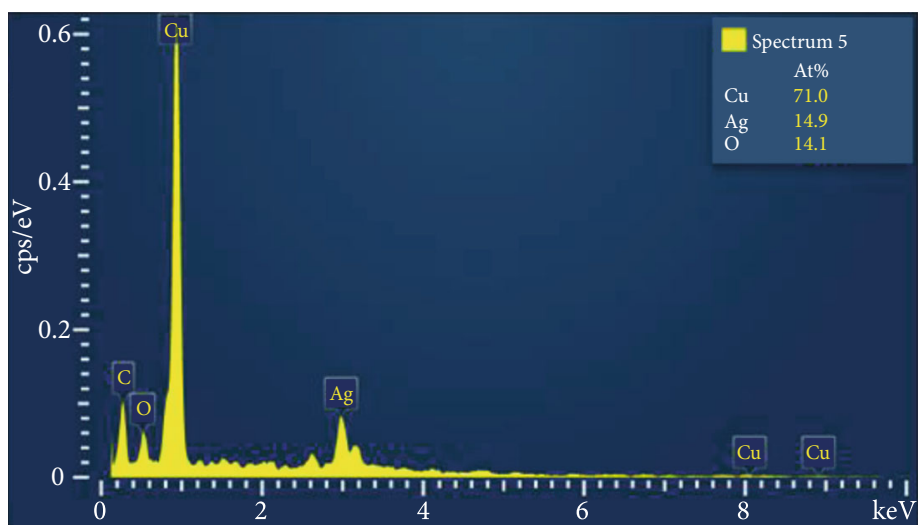

(c)

Figure 2: (a) XRD pattern, (b) TEM image, and (c) EDX spectrum of the biogenic AgNPs synthesized at $12 \mathrm{~V}$. 


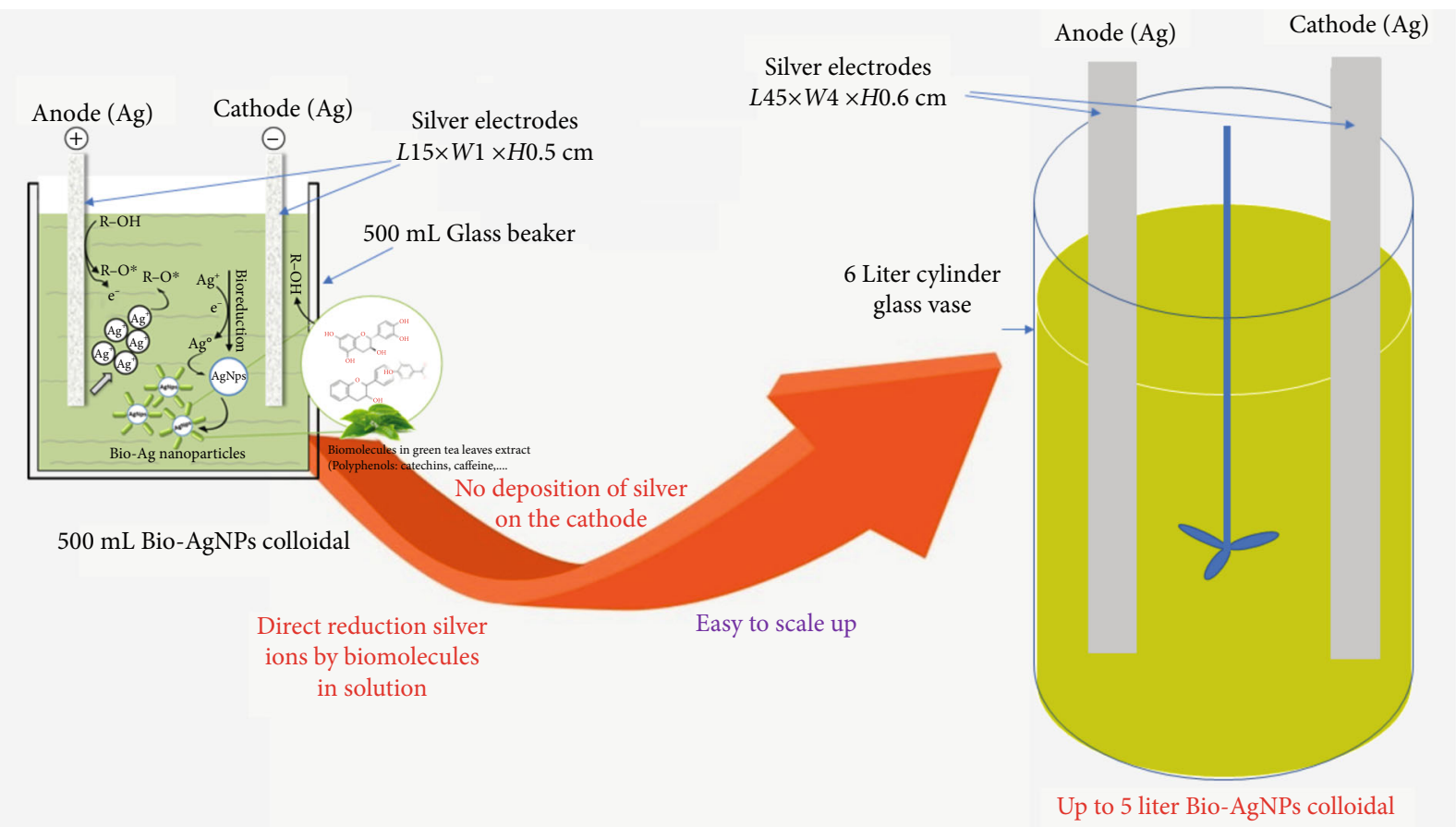

FIGURE 3: Schematic representation of the biogenic AgNPs' scale-up generation mechanism by using both electrochemical process and GTE as the reducing agent.

Additionally, the effect of the amount of green tea on the yield of biogenic AgNPs was also evaluated using UV-vis spectroscopy. Figure 1(b) shows the UV-vis absorption spectra of biogenic AgNPs prepared using various amounts of GTE. It was also observed that the intensity of SPR band at $437 \mathrm{~nm}$ obviously increased with increasing amount of green tea and reached maximum at $2 \mathrm{~g}$. The increase of characteristic band intensity is attributed to the increasing number of nanoparticles formed as a result of greater releasing of $\mathrm{Ag}^{+}$ ions from Ag anode at higher GTE concentration [7, 13]. These results indicate that the amount of green tea can affect the yield of biogenic AgNPs. However, when the amount of green tea is $2 \mathrm{~g}$, the further increase of number of $\mathrm{Ag}^{+}$ions and the released oxygen can increase the formation of $\mathrm{Ag}_{2} \mathrm{O}$ leads to the yield of biogenic AgNPs is decreased.

The formation of biogenic AgNPs was further confirmed by X-ray and TEM measurements. XRD analysis and TEM results of biogenic AgNPs were shown in Figure 2. In Figure 2(a), it is clear to see three diffraction peaks at $38.1^{\circ}$, $44.3^{\circ}$, and $64.4^{\circ}$ correspond to the $\mathrm{d}(111), \mathrm{d}(200)$, and $\mathrm{d}(220)$ fcc crystal planes of bulk Ag (JCPDS No. 04-0783), respectively. The TEM observation also reveals that assynthesized biogenic AgNPs were spherical, homogeneous in size range of $34 \mathrm{~nm}$, and shape. As shown in Figure 2(b), it can be seen that the presence of other material membrane around silver nanoparticles might be due to the formation of biomolecule matrix from GTE. The EDX spectrum of the biogenic AgNPs (Figure 2(c)) reveals the existence of Ag, $\mathrm{O}$, and $\mathrm{C}$ components in the sample. The existence of $\mathrm{O}$ and $\mathrm{C}$ components can be due to emission from the formation of biomolecule matrix around AgNPs. In addition, EDX spectrum confirmed a high atomic weight percentage of $\mathrm{Cu}$ element, due to emission of the copper substrate used in the SEM and EDX measurements. These results exhibited that novel biogenic AgNPs were successfully synthesized by using both green electrochemical method and GTE extracts as reducing agent.

3.2. The Formation Mechanism of Biogenic AgNPs by GTEMediated Green Electrochemical Method. As described in Figure 3 and according to some previous reports, under a direct-current voltage source, the electron transfer occurred at the anode side, biomolecules in GTE (R-OH) were decreased to form R-O. radicals and release electrons [14]. Then, $\mathrm{Ag}^{+}$ions, which were released from the anode, interacted with R-O. radicals and received electrons to form $\mathrm{Ag}^{0}$ atoms. As a result, the silver atoms then grew to form the biogenic AgNPs. Here, the biomolecules played an essential role in both reductant and stabilizer for AgNPs. Stabilization may likely be caused by capping of natural biomolecules such as the flavonoid and terpenoid components to create a monolayer on the AgNPs surface in solution. On the other hand, the formation of AgNPs on the cathode side as explained by Khaydarov et al. [6] could be taken place, but it was remarkably prevented due to the formation of $\mathrm{R}-\mathrm{OH}-\mathrm{Ag}^{+}$ and stirring force of a magnetic stirrer. The processes of the AgNP formation are described below [6, 13-15].

(1) The formation of R-O. radicals and releasing of $\mathrm{Ag}^{+}$ ions from $\mathrm{Ag}$ anode:

$$
\begin{gathered}
\mathrm{R}-\mathrm{OH} \rightarrow \mathrm{R}-\mathrm{O}+\mathrm{H}^{+}+\mathrm{e}^{-} \\
\mathrm{Ag}^{0}-\mathrm{e}^{-} \rightarrow \mathrm{Ag}^{+}
\end{gathered}
$$



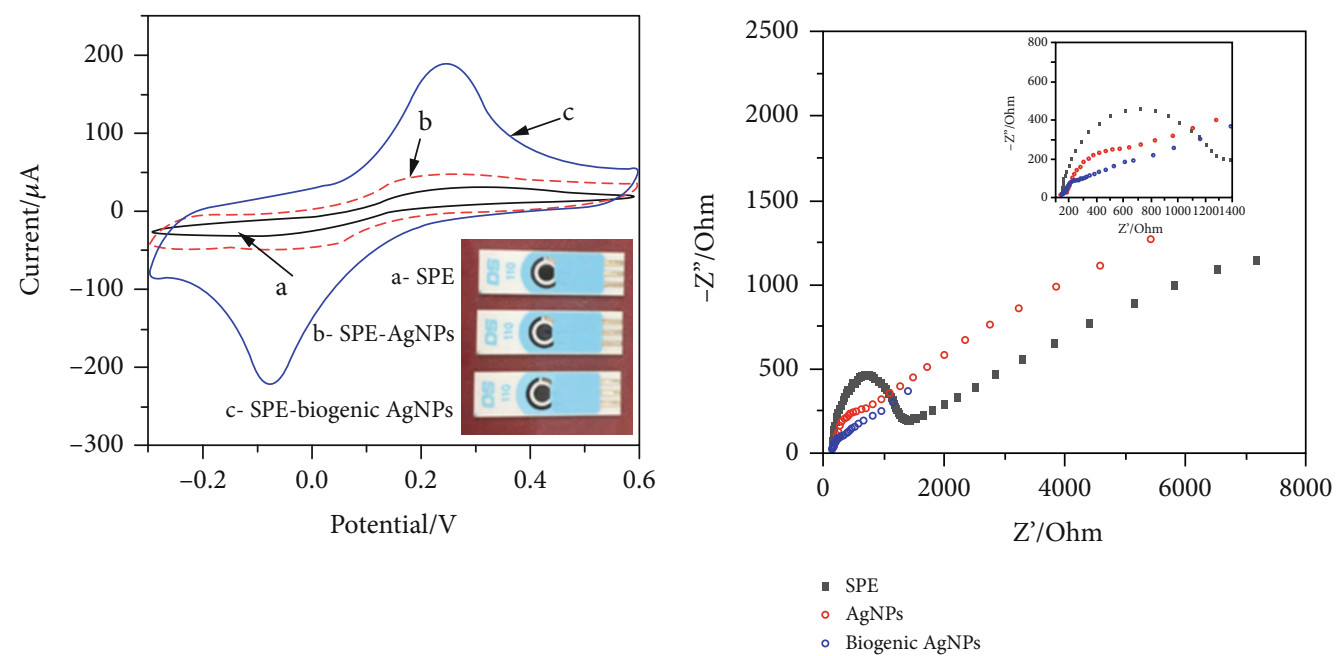

(a)

(b)

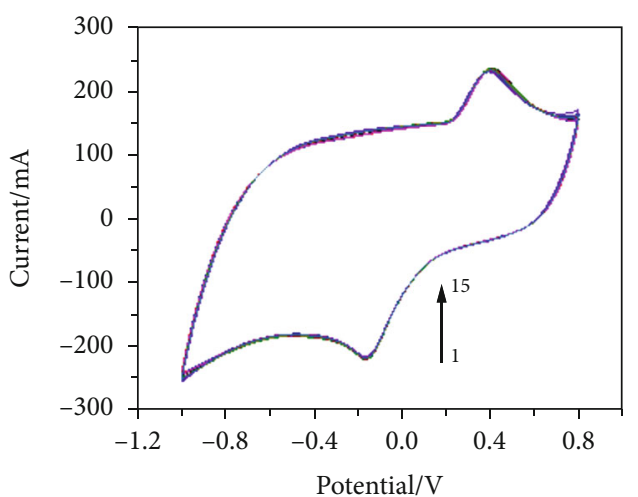

(c)

Figure 4: (a) CV of bare SPE (curve a), pure AgNPs-SPE (curve b), and biogenic AgNP-SPE (curve c) electrodes in 5 mM $\mathrm{K}_{3}\left[\mathrm{Fe}(\mathrm{CN})_{6}\right] / \mathrm{K}_{4}\left[\mathrm{Fe}(\mathrm{CN})_{6}\right](1: 1)$ mixture at $50 \mathrm{mV} \mathrm{s}^{-1}$; (b) Nyquist plots of bare SPE, pure AgNPs-SPE, and biogenic AgNP-SPE electrodes recorded in $0.1 \mathrm{~mol} \mathrm{~L}^{-1} \mathrm{KCl}$ solution containing $5 \mathrm{mM} \mathrm{K}_{3}\left[\mathrm{Fe}(\mathrm{CN})_{6}\right] / \mathrm{K}_{4}\left[\mathrm{Fe}(\mathrm{CN})_{6}\right]$; (c) CV of biogenic AgNP-SPE electrode for 15 cycles.

(2) The formation of the intermediate complex:

$$
\mathrm{R}-\mathrm{O}+\mathrm{Ag}^{+} \rightarrow \mathrm{R}-\mathrm{O}---\mathrm{Ag}^{+}
$$

(3) Reductive formation of $\mathrm{Ag}^{0}$ atom in solution:

$$
\mathrm{R}-\mathrm{O}+\mathrm{Ag}^{+}+\mathrm{e}^{-} \rightarrow \mathrm{R}=\mathrm{O}(\text { quinone })+\mathrm{Ag}^{0}
$$

Role of the -OH groups in the bioreduction of $\mathrm{Ag}^{+}$to $\mathrm{Ag}^{0}$ and the formation of AgNPs was discussed by Hussain and Khan [16]. It is easy to transfer the proton to $\mathrm{Ag}^{+}$ions to form AgNPs under an applied voltage condition. Moreover, the formation of the intermediate complex $\left(\mathrm{R}-\mathrm{O}-\mathrm{Ag}^{+}\right)$not only enhances the formed AgNP stabilization [17] and protects them from the oxidation arising from the release of oxygen or hydrogen due to the electrolysis of water in GTE, but also prevents the reduction of $\mathrm{Ag}^{+}$ions at the surface of the cathode. Thus, the technological yield of AgNP synthesis process was significantly improved compared to some conventional electrochemical methods. The synthesis yield of AgNP formation calculated is about $98 \%$.
In the synthesis of biogenic AgNPs using normal plant extracts, biogenic AgNP production was affected by many other factors such as the reducing capacity of the biomolecules, extract dosage, reaction time, and temperature [4, 16]. For the synthesis of biogenic AgNPs using GTE at room temperature and pressure, the preparation time from $2 \mathrm{~h}$ to $24 \mathrm{~h}$ was needed $[17,18]$. The reduction process in a long time causes poor crystallization of AgNPs, low efficiency, and difficulty in the large scale-up produce [19]. In fact, some recent reports show that the biogenic AgNP formation process was promoted and controlled by using high temperature [13], microwave [4], and hydrothermal [20]. Instead of that, in this study, the utilization of the direct current contributes to promote the electron releasing process and the formation of R-O. radicals. According to that, the reducing process of $\mathrm{Ag}^{+}$into $\mathrm{Ag}^{0}$ induced more quickly, leading to improve the quality of biogenic AgNP nanocrystals. It should be emphasized that the above-proposed method not only offers many promising benefits for biogenic AgNPs synthesis in the future such as lowcost, without using chemical additives, rapid synthesis, and simple scale-up but also uses the herb resources from nature. The technological keys to the electrochemical 


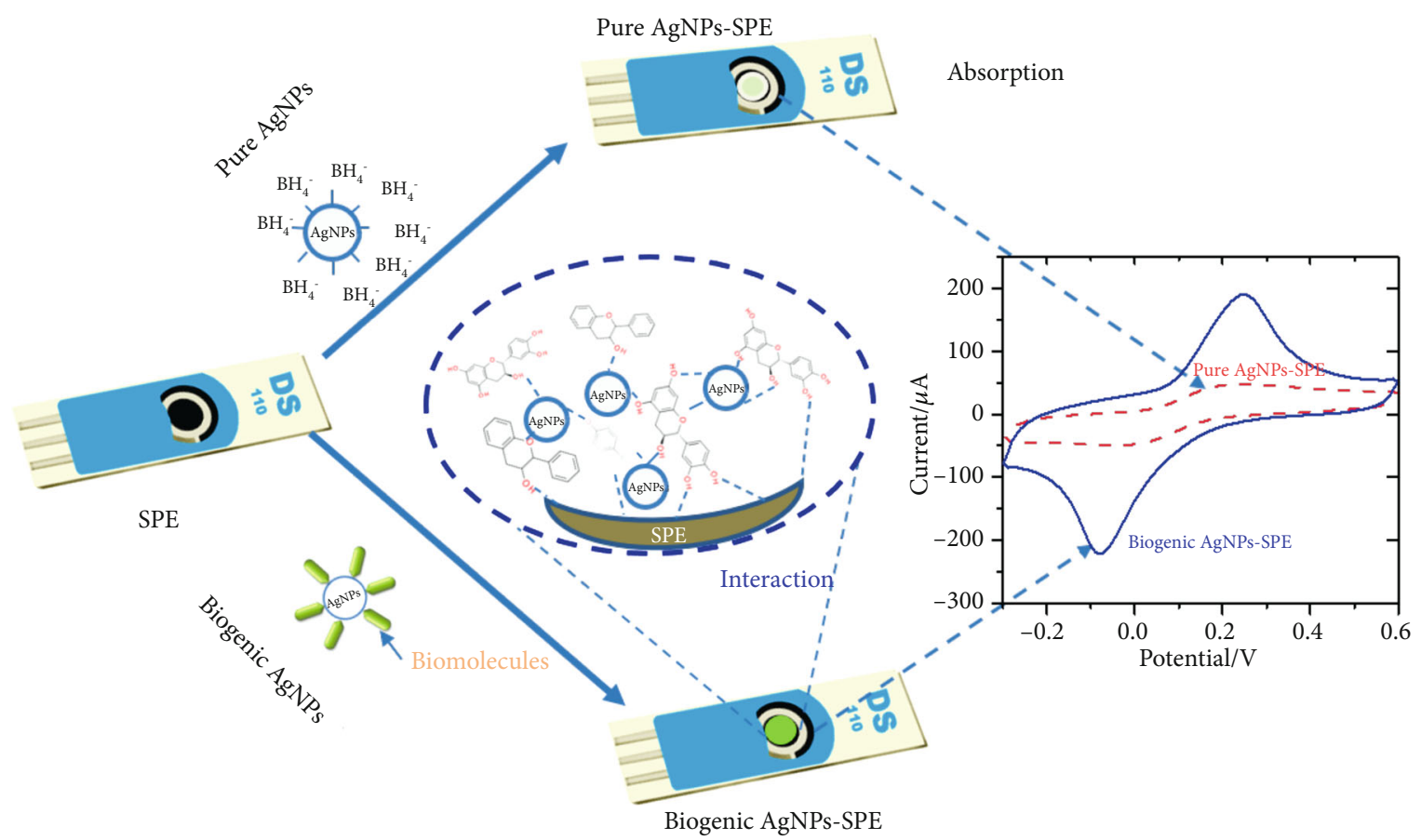

FIGURE 5: A mechanism for the enhanced electrochemical properties of the biogenic AgNPs deposited on the surface of the SPE compared to the pure AgNPs.

synthesis of biogenic AgNPs are the voltage value applied on two electrodes in green tea leave extract.

3.3. The Electrochemical Activity of Novel Biogenic AgNPs. The electrochemical activity of novel biogenic AgNPs was analyzed and compared to pure AgNPs using two electrochemical techniques, including the $\mathrm{CV}$ and EIS techniques. We evaluated the charge transfer performance of asprepared samples, and the CV of bare SPE, pure AgNPsSPE, and biogenic AgNP-SPE electrodes was recorded in a mixture of $5 \mathrm{mM} \mathrm{K}_{3}\left[\mathrm{Fe}(\mathrm{CN})_{6}\right] / \mathrm{K}_{4}\left[\mathrm{Fe}(\mathrm{CN})_{6}\right](1: 1)$ at a scan rate of $50 \mathrm{mV} \mathrm{s}^{-1}$ as shown in Figure 4(a). For the bare SPE, the cyclic voltammetry response displayed the low current signal $\left(I_{a}=28 \mu \mathrm{A}\right.$ and $\left.I_{c}=-26 \mu \mathrm{A}\right)$. The $\mathrm{CV}$ response was improved for pure AgNPs-SPE $\left(I_{a}=30 \mu \mathrm{A}\right.$ and $I_{c}=-43$ $\mu \mathrm{A})$, suggesting that pure AgNPs were decorated on the SPE surface. More interestingly, compared to the bare SPE and pure AgNPs-SPE, biogenic AgNPs-SPE exhibited the outstanding current signal with $I_{a}=137 \mu \mathrm{A}$ and $I_{c}=-182$ $\mu \mathrm{A}$. This current signal enhancement can be due to the excellent conductivity and the large specific surface area of uniform biogenic AgNPs, as well as the formation of biomolecule matrix around AgNPs.

Figure 4(b) illustrates that the Nyquist plots of bare SPE, pure AgNPs-SPE, and biogenic AgNP-SPE electrodes were recorded in $0.1 \mathrm{~mol} \mathrm{~L}^{-1} \mathrm{KCl}$ solution containing $5 \mathrm{mM}$ $\mathrm{K}_{3}\left[\mathrm{Fe}(\mathrm{CN})_{6}\right] / \mathrm{K}_{4}\left[\mathrm{Fe}(\mathrm{CN})_{6}\right]$. As can be observed, the Nyquist plots of all electrodes displayed a semicircle portion at higher frequencies to a limited electron transfer process and a linear part at a lower frequency range corresponding to the diffusion process. For the bare SPE, the calculated value of electron-transfer resistance $\left(R_{\mathrm{ct}}\right)$ from diameter of semicircle was about $1109 \Omega$ (Figure 4(b), black curve). After bare SPE was modified with pure AgNPs, the value of $R_{\text {ct }}$ decreased to $504 \Omega$. Particularly, compared with pure AgNPs-SPEs, the EIS of the biogenic AgNPs-SPEs showed smaller semicircular regions with a $R_{\mathrm{ct}}$ value of approximately $140 \Omega$. The lower $R_{\mathrm{ct}}$ value for biogenic AgNPs-SPEs indicates that the electron transport rate between the medium and electrode was significantly increased upon the SPE modification with biogenic AgNPs, which can be due to the effect of biomolecule matrix in the ability of modified electrode. These results also corroborated well the obtained results from CV measurements. More particularly, the electrochemical stability performance of biogenic AgNP-SPE electrode was also examined. As shown in Figure 4(c), after 15 cycles, the change of the reduction peak currents in CV measurements only varied $\sim 2.4 \%$, indicating that the active electrode surface and the immobilization of biogenic AgNPs on the SPE surface were extremely stable. The mechanism for the enhanced electrochemical properties of biogenic AgNPs deposited on the surface of the SPE as compared with pure AgNPs was described in Figure 5. This enhancement can be attributed due to the attachment of biogenic AgNPs on the carbon surface of the SPE through the formation of the $\mathrm{C}-\mathrm{O}$ coordinate bonds between $\mathrm{O}$ atom of biomolecules on the biogenic AgNPs surface and C atom of SPE [21, 22]. The bonding formation of biogenic AgNPs with SPE based on biomolecules as a linker helps enhance the electron transfer kinetics and stability during the electrochemical process. Besides, the presence of biomolecule matrixes owning many flexible $\pi-\pi$ bonds around AgNPs also facilitates the rapid electron transfer and enhances the active surface area. In contrast, for pure AgNPs-SPE, AgNPs which were stabilized 


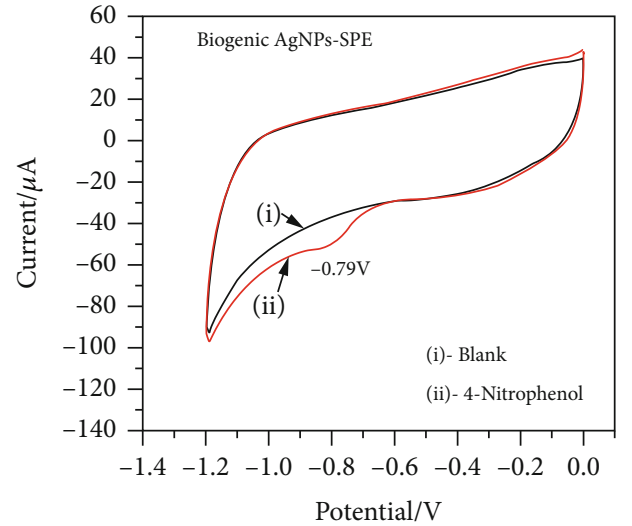

(a)

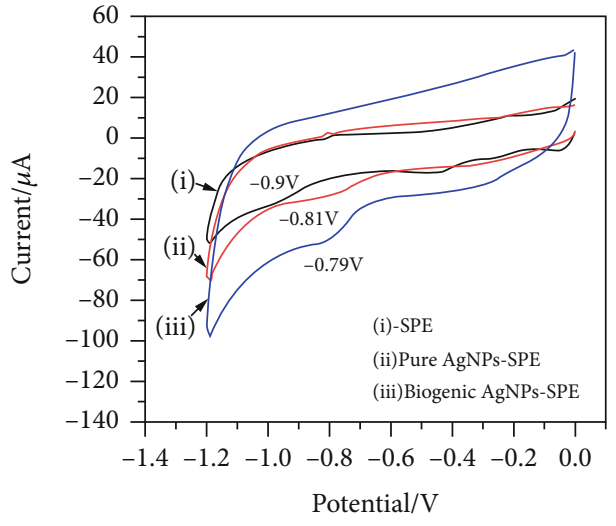

(b)

Figure 6: CV of (a) $10 \mu \mathrm{M}$ 4-NP at biogenic AgNPs-SPE in 0.1 M PBS (pH 7.4); (b) $10 \mu \mathrm{M}$ of 4-NP at bare SPE, pure AgNPs-SPE, and biogenic AgNPs-SPE in 0.1 M PBS (pH 7.4).

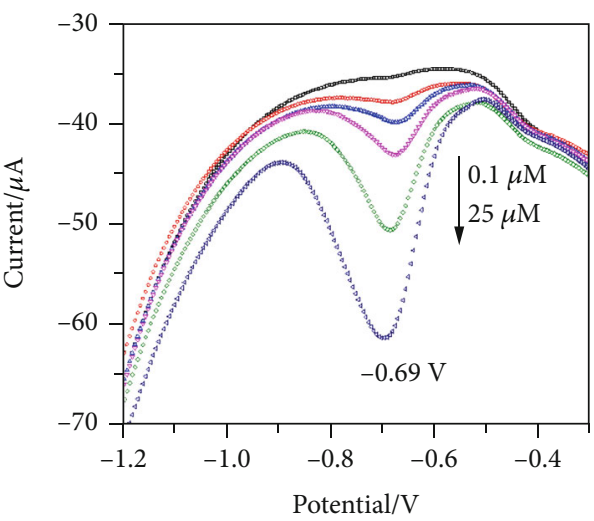

(a)

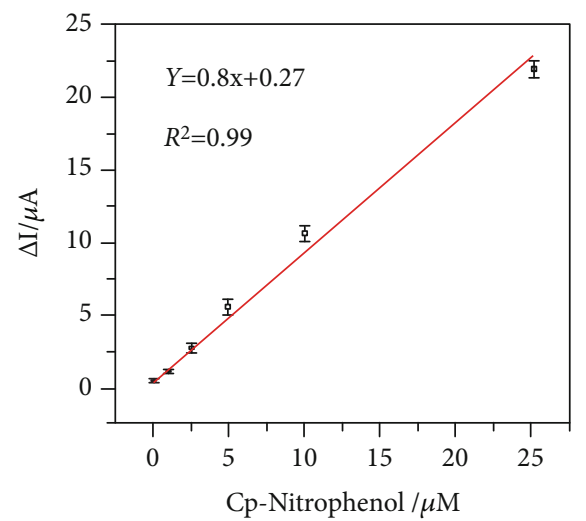

(b)

FIGURE 7: DPV in 0.1 M PBS (pH 7.4) containing different concentrations of 4-NP: (a) biogenic AgNPs-SPE for 4-NP at different concentrations and (b) linear relationship between peak currents and 4-NP concentrations.

by $\mathrm{BH}_{4}{ }^{-}$are only absorbed physically on the SPE surface without bonding agents. More importantly, biogenic AgNP-SPE is able to easily and strongly adsorb the analytes such as ions, chemical compounds due to the conjugation effect and electrostatic interaction of biomolecules, along with that the oxygen functional groups can improve the electrocatalytic activity of the synthesized electrode [23]. These results suggest that the biogenic AgNPs-SPE will be an ideal candidate for green electrochemical sensors in a variety of environmental monitoring and biomedical applications.

3.4. The Use of Biogenic AgNPs as Probe for Detection of 4Nitrophenol. In the following, to demonstrate further the promising electrochemical properties of the biogenic AgNPs-SPE, the 4-NP detection capability using the SPE, pure AgNPs-SPE, and biogenic AgNPs-SPE electrodes was performed (Figure 6) in 0.1 M PBS (pH 7.4). Figure 6(a) shows the CV response of the biogenic AgNPs-SPE in $0.1 \mathrm{M}$ PBS (pH 7.4) without (curve i) and with $10 \mu \mathrm{M} 4-\mathrm{NP}$ (curve ii). In the case adding of 4-NP, there was a sharp peak at $-0.79 \mathrm{~V}$, which is good agreement with some previous reports related to the reduction peak of 4-NP [10-12].

Figure 6(b) shows the CV response of $10 \mu \mathrm{M}$ of $4-\mathrm{NP}$ in $0.1 \mathrm{M}$ PBS (pH 7.4) for all the three electrodes. The results show that it was observed at the reduction peak of 4-NP at $-0.9 \mathrm{~V}$ (curve i), $-0.81 \mathrm{~V}$ (curve ii), and $-0.79 \mathrm{~V}$ (curve iii) in the case of the SPE, pure AgNPs-SPE, and biogenic AgNPsSPE, respectively. The pure AgNPs-SPE and biogenic AgNPs-SPE showed the higher catalytic current responses than that of bare SPE. This result indicates that AgNPmodified electrodes possess good electrocatalytic ability towards 4-NP, due to the good conductivity and compatibility of AgNPs [8, 12]. Additionally, the biogenic AgNPs-SPE showed the enhancement of current signal when compared to pure AgNPs-SPE because of the presence of the biomolecule matrix. This enhancement is a positive result arising from the strong electrostatic interaction and new bindings formed between biomolecules and 4-NP as proposed above.

Figure 7(a) exhibits the differential pulse voltammetry (DPV) response of the biogenic AgNPs-SPE for the variation in the concentrations of 4-NP at the scan rate of $6 \mathrm{mV} \mathrm{s}^{-1}$ and 
the potential scanning from $-0.4 \mathrm{~V}$ to $-1.2 \mathrm{~V}$. In the concentration range of $0.1-25 \mu \mathrm{M}$, the obtained calibration curves (Figure 7(b)) show that there was a linear relationship between the electrochemical responses and 4-NP concentrations with the correlation coefficient, $R^{2}=0.99$. The sensitivity for this electrode was found to be $6.69 \mu \mathrm{A} \mu \mathrm{M}^{-1} \mathrm{~cm}^{-2}$. From this observation, it is clear that the utilization of biogenic AgNPs as electrocatalyst created a positive effect on electron transfer process during the redox reaction of 4-NP; furthermore, it played an essential role as great electrocatalyst for the reduction of $4-\mathrm{NP}$.

\section{Conclusions}

A scalable green electrochemical method has been presented for the rapid and large synthesis of biogenic AgNPs using the bulk silver bars and GTE as reducing agent, along with a direct-current voltage source. The proposed method was based on a simple electrochemical process that can rapidly synthesize the purity biogenic AgNPs with high yield, notably, without the use of any additive chemicals. Interestingly, the as-synthesized biogenic AgNPs with an average particle size of $34 \mathrm{~nm}$ were applied as an excellent probe for the 4NP detection. The biogenic AgNPs-SPE demonstrated good sensitivity $\left(6.69 \mu \mathrm{A} \mu \mathrm{M}^{-1} \mathrm{~cm}^{2}\right)$ in the linear range from $0.1 \mu \mathrm{M}$ to $25 \mu \mathrm{M}$ of $4-\mathrm{NP}$, suggesting that biogenic AgNPs are the ideal candidates for green electrochemical sensors. It can be believed that the biogenic AgNPs will be a promising material for biomedical and bio/chemosensing applications.

\section{Data Availability}

Data are available on request.

\section{Conflicts of Interest}

The authors declare that they have no conflicts of interest.

\section{Authors' Contributions}

V.T. Hoang and N.X. Dinh contributed equally to this work.

\section{Acknowledgments}

This research was funded by Phenikaa University under grant number 02.2019.03.

\section{References}

[1] Y. A. Krutyakov, A. A. Kudrinskiy, A. Y. Olenin, and G. V. Lisichkin, "Synthesis and properties of silver nanoparticles: advances and prospects," Russian Chemical Reviews, vol. 77, no. 3, pp. 233-257, 2008.

[2] B. Zewde, A. Ambaye, J. Stubbs Iii, and D. Raghavan, "A review of stabilized silver nanoparticles-synthesis, biological properties, characterization, and potential areas of applications," Nanomed, vol. 4, p. 1043, 2016.

[3] Q. H. Tran, V. Q. Nguyen, and A.-T. Le, "Silver nanoparticles: synthesis, properties, toxicology, application and perspec- tives," Advances in Natural Sciences: Nanoscience and Nanotechnology, vol. 4, article 033001, 2013.

[4] M. Sökmen, S. Y. Alomar, C. Albay, and G. Serdar, "Microwave assisted production of silver nanoparticles using green tea extracts," Journal of Alloys and Compounds, vol. 725, pp. 190-198, 2017.

[5] W. R. Rolim, M. T. Pelegrino, B. de Araújo Lima et al., "Green tea extract mediated biogenic synthesis of silver nanoparticles: characterization, cytotoxicity evaluation and antibacterial activity," Applied Surface Science, vol. 463, pp. 66-74, 2019.

[6] R. A. Khaydarov, R. R. Khaydarov, O. Gapurova, Y. Estrin, and T. Scheper, "Electrochemical method for the synthesis of silver nanoparticles," Journal of Nanoparticle Research, vol. 11, no. 5, pp. 1193-1200, 2009.

[7] D. T. Thuc, T. Q. Huy, L. H. Hoang et al., "Green synthesis of colloidal silver nanoparticles through electrochemical method and their antibacterial activity," Materials Letters, vol. 181, pp. 173-177, 2016.

[8] C. Karuppiah, S. Palanisamy, S.-M. Chen et al., "Green biosynthesis of silver nanoparticles and nanomolar detection of pnitrophenol," Journal of Solid State Electrochemistry, vol. 18, no. 7, pp. 1847-1854, 2014.

[9] M. Mavaei, A. Chahardoli, Y. Shokoohinia, A. Khoshroo, and A. Fattahi, "One-step synthesized silver nanoparticles using isoimperatorin: evaluation of photocatalytic, and electrochemical activities," Scientific Reports, vol. 10, no. 1, p. 1762, 2020.

[10] C. Zhang, S. Govindaraju, K. Giribabu, Y. S. Huh, and K. Yun, "AgNWs-PANI nanocomposite based electrochemical sensor for detection of 4-nitrophenol," Sensors and Actuators B: Chemical, vol. 252, pp. 616-623, 2017.

[11] B. Dinesh and R. Saraswathi, "Electrochemical synthesis of nanostructured copper-curcumin complex and its electrocatalytic application towards reduction of 4-nitrophenol," Sensors and Actuators B: Chemical, vol. 253, pp. 502-512, 2017.

[12] N. I. Ikhsan, P. Rameshkumar, and N. M. Huang, "Controlled synthesis of reduced graphene oxide supported silver nanoparticles for selective and sensitive electrochemical detection of 4nitrophenol," Electrochimica Acta, vol. 192, pp. 392-399, 2016.

[13] M. R. Siddiqui, M. Khan, A. Khan, W. T. Tahir, and W. Khathlan, "Green synthesis of silver nanoparticles mediated by Pulicaria glutinosa extract," International Journal of Nanomedicine, vol. 1507, 2013.

[14] S. R. Waldvogel, S. Mentizi, and A. Kirste, "Boron-doped diamond electrodes for electroorganic chemistry," in Radicals in Synthesis IIIpp. 1-31, Springer, Berlin, Heidelberg.

[15] N. Tarannum, D. Divya, and Y. K. Gautam, "Facile green synthesis and applications of silver nanoparticles: a state-of-theart review," RSC Advances, vol. 9, no. 60, pp. 34926-34948, 2019.

[16] S. Hussain and Z. Khan, "Epigallocatechin-3-gallate-capped Ag nanoparticles: preparation and characterization," Bioprocess and Biosystems Engineering, vol. 37, no. 7, pp. 12211231, 2014.

[17] A. Gade, S. Gaikwad, V. Tiwari, A. Yadav, A. Ingle, and M. Rai, "Biofabrication of silver nanoparticles by Opuntia ficus-indica: in vitro antibacterial activity and study of the mechanism involved in the synthesis," Current Nanoscience, vol. 6, no. 4, pp. 370-375, 2010.

[18] Q. Sun, X. Cai, J. Li, M. Zheng, Z. Chen, and C.-P. Yu, “Green synthesis of silver nanoparticles using tea leaf extract and evaluation of their stability and antibacterial activity," Colloids and 
Surfaces A: Physicochemical and Engineering Aspects, vol. 444, pp. 226-231, 2014.

[19] M. A. Asghar, E. Zahir, S. M. Shahid et al., "Iron, copper and silver nanoparticles: green synthesis using green and black tea leaves extracts and evaluation of antibacterial, antifungal and aflatoxin B1 adsorption activity," LWT, vol. 90, pp. 98107, 2018.

[20] P. Tippayawat, N. Phromviyo, P. Boueroy, and A. Chompoosor, "Green synthesis of silver nanoparticles in aloe vera plant extract prepared by a hydrothermal method and their synergistic antibacterial activity," PeerJ, vol. 4, article e2589, 2016.

[21] V. Kumar, R. K. Gupta, R. K. Gundampati et al., "Enhanced electron transfer mediated detection of hydrogen peroxide using a silver nanoparticle-reduced graphene oxide-polyaniline fabricated electrochemical sensor," RSC Advances, vol. 8, p. 619,2018

[22] J. Simonet, "Enolization versus carbonylation at glassy carbon surface through cathodic means," Electrochemistry Communications, vol. 31, pp. 1-4, 2013.

[23] T. Sun, H. Pan, Y. Mei et al., "Electrochemical sensor sensitive detection of chloramphenicol based on ionic-liquid-assisted synthesis of de-layered molybdenum disulfide/graphene oxide nanocomposites," Journal of Applied Electrochemistry, vol. 49, no. 3, pp. 261-270, 2019. 